\title{
Refined inversion statistics on permutations
}

\author{
Joshua Sack ${ }^{*}$ \\ Department of Mathematics and Statistics \\ California State University, Long Beach, USA \\ joshua.sack@gmail.com
}

\author{
Henning Úlfarsson ${ }^{\dagger}$ \\ School of Computer Science \\ Reykjavik University, Iceland \\ henningu@ru.is
}

Submitted: Jul 10, 2011; Accepted: Jan 12, 2012; Published: Jan 27, 2012

Mathematics Subject Classification: 05A05

\begin{abstract}
We introduce and study new refinements of inversion statistics for permutations, such as $k$-step inversions, (the number of inversions with fixed position differences) and non-inversion sums (the sum of the differences of positions of the non-inversions of a permutation). We also provide a distribution function for non-inversion sums, a distribution function for $k$-step inversions that relates to the Eulerian polynomials, and special cases of distribution functions for other statistics we introduce, such as $(\leq k)$-step inversions and $\left(k_{1}, k_{2}\right)$-step inversions (that fix the value separation as well as the position). We connect our refinements to other work, such as inversion tops that are 0 modulo a fixed integer $d$, left boundary sums of paths, and marked meshed patterns. Finally, we use non-inversion sums to show that for every number $n>34$, there is a permutation such that the dot product of that permutation and the identity permutation (of the same length) is $n$.
\end{abstract}

\section{Contents}

1 Introduction $\quad 2$

2 Non-inversion sums and the dot product of permutations $\quad 3$

3 Zone-crossing vectors and the distribution of the non-inversion sum 10

4 The distribution of $k$-, $\left(k_{1}, k_{2}\right)$-, and $(\leq k)$-step inversions $\quad 14$

5 Future work and connections with other work $\quad 21$

*Sack was partly supported by grant no. 100048021 from the Icelandic Research Fund.

†Úlfarsson was supported by grant no. 090038011 from the Icelandic Research Fund. 


\section{Introduction}

The main object of study in this paper is the set of inversions in a permutation. ${ }^{1}$ An inversion in a permutation $\pi$, of rank $n$, is a pair $(a, b)$ satisfying $1 \leq a<b \leq n$ and $\pi(a)>\pi(b)$. All other pairs are called non-inversions. We are particularly interested in permutation statistics related to inversions, such as the number of inversions of a certain form. The study of permutation statistics was largely initiated by the seminal MacMahon [6], but has seen explosive growth in recent decades. In Section 2 we introduce the concept of the non-inversion sum of a permutation. This is the sum of the differences $b-a$ for all non-inversions $(a, b)$ in the permutation. Before studying the distribution of this statistic we connect these non-inversion sums to another known statistic on permutations: the dot product with a fixed vector. In particular, the dot product of the permutation (treated as a vector) with the identity permutation of the same length is equal to the non-inversion sum of the permutation plus a function of the rank of the permutation; see Theorem 2.5.

In Section 3, we define the distribution function for the non-inversion sum and prove a recurrence relation for it in Theorem 3.8. We introduce the concept of a zone-crossing vector, which appears in the recurrence relations. This is a vector whose $k^{\text {th }}$ coordinate is the number of non-inversions $(a, b)$ such that $a \leq k<b$. We relate these vectors to the non-inversion sums and show that there is a bijective correspondence between permutations and their zone-crossing vectors. We also prove a theorem showing that the distribution of the coordinates of these vectors is related to the $q$-analog of the binomial coefficients; see Theorem 3.7.

In Section 4 we consider $k$-step inversions, which are inversions $(a, b)$ such that $b-a=$ $k$, and show in Theorem 4.4 that the distribution of these types of inversions is related to the Eulerian polynomials. We next consider $\left(k_{1}, k_{2}\right)$-step inversions, which are inversions $(a, b)$, such that $b-a=k_{1}$ and $\pi(b)-\pi(a)=k_{2}$, and prove a special case of the distribution function; see Proposition 4.6. We also consider inversions $(a, b)$ such that $b-a \leq k$ and prove recurrence relations for their distributions in some special cases; see Proposition 4.8.

In Section 5, we consider some relationships between our work and the work of others. In Section 5.1, we consider a $k$-step variant of a statistic that counts inversions whose first coordinate (called the inversion top) is 0 modulo $d$. Inversion tops mod $d$ have been studied by Kitaev and Remmel [4, 5] and by Jansson [3]. We provide formulas for special cases of the distribution of $k$-step inversions whose first coordinate is $0 \bmod d$.

In Section 5.2, we consider a $k$-step variant of the left boundary sums in Dukes and Reifergerste [2]. Given a permutation $\pi$, the left boundary sum of $\pi$ (denoted Ibsum $(\pi)$ ) gives the area to the left of the Dyck path of $\pi$. Dukes and Reifergerste [2] show that $\operatorname{Ibsum}(\pi)$ is also the sum of the number of inversions and the number of certified noninversions, where a certified non-inversion is a non-inversion $(a, b)$, with a position $c$, such that $a<c<b$ and $\pi_{c} \geq \pi_{d}$ whenever $a<d<b$. We consider a $k$-step variant of this (denoted $\operatorname{ipcni}_{k}(\pi)$ ) that only counts $k$-step inversions and $k$-step certified non-inversions, and provide special cases of the distribution functon. Finally we show how many of the

\footnotetext{
${ }^{1}$ We provide basic definitions at the end of this introduction.
} 
statistics we consider can be represented using marked mesh patterns defined by Úlfarsson in $[7]$

The connection found in Theorem 2.5 is used in Theorem 2.2 to show that given any integer $k$ greater than 34 there exists a permutation $\pi$ such that the dot product of $\pi$ with the identity permutation $12 \cdots|\pi|$ equals $k$. We also present an algorithm that, given $k$, produces the permutation $\pi$; see Section 2.1. The total number of permutations which dotted with the identity permutation gives $k$, is given by the sequence A $135298^{2}$ in the Online Encyclopedia of Integer Sequences, and hence our theorem tells us that this sequence is non-zero after $k=34$.

\section{Basic definitions}

We define the set of positive integers to be $\mathbb{P}=\{1,2,3, \ldots\}$. A permutation is a bijective function $\pi:\{1, \ldots, n\} \rightarrow\{1, \ldots, n\}$ for some $n$ in $\mathbb{P}$. The number $n$ is called the rank of the permutation. We often write $\pi_{k}$ for $\pi(k)$, and write a permutation as a list of its values $\pi_{1} \pi_{2} \cdots \pi_{n}$. Let $\mathfrak{S}_{n}$ be the set of permutations of rank $n$.

We define the identity permutation $\mathbf{1}_{n}$ as the permutation $\pi$, such that $\pi_{k}=k$ for $1 \leq k \leq n$. We will write $\mathbf{1}$, omitting the subscript, if the rank is clear from the context. Given a permutation $\pi=\pi_{1} \pi_{2} \cdots \pi_{n}$, we define its reverse as $\pi^{\mathrm{r}}=\pi_{n} \pi_{n-1} \cdots \pi_{1}$, its complement as $\pi^{\mathrm{c}}=\left(n+1-\pi_{1}\right)\left(n+1-\pi_{2}\right) \cdots\left(n+1-\pi_{n}\right)$, and its inverse $\pi^{\mathrm{i}}$ as the unique permutation such that $\pi \circ \pi^{\mathrm{i}}=\mathbf{1}$.

\section{Non-inversion sums and the dot product of permu- tations}

Definition 2.1. For a permutation $\pi$ of rank $n$, the number

$$
\mathbf{1} \cdot \pi=\sum_{i=1}^{n} i \pi(i)
$$

is called the cosine of the permutation.

Note that if we treat permutations as vectors then

$$
\mathbf{1} \cdot \pi=|\mathbf{1}| \cdot|\pi| \cos (\theta)=\left(1^{2}+2^{2}+\cdots+n^{2}\right) \cos (\theta)=\frac{n(n+1)(2 n+1)}{6} \cos (\theta),
$$

where $\theta$ is the angle between $\mathbf{1}$ and $\pi$. So $\mathbf{1} \cdot \pi$ only depends on the cosine of the angle between the identity and the permutation.

Most of this section will be leading to a proof of the following theorem:

Theorem 2.2. For a positive integer

$$
k \notin\{2,3,6,7,8,9,12,15,16,17,18,19,31,32,33,34\},
$$

there exists a permutation $\pi$ such that $\mathbf{1} \cdot \pi=k$.

\footnotetext{
${ }^{2}$ http://oeis.org/A135298
} 
The total number of permutations $\pi$, such that $\mathbf{1} \cdot \pi=k$, is given by the sequence A $135298^{3}$ in the Online Encyclopedia of Integer Sequences. Our theorem tells us that this sequence is non-zero after $k=34$. Furthermore, we will provide an algorithm in Section 2.1 for constructing a permutation $\pi$, such that $\mathbf{1} \cdot \pi=k$ for $k$ as in the theorem.

To prove this theorem, we introduce the notion of the non-inversion sum. We build this notion on that of a non-inversion. Given a permutation $\pi$ of rank $n$, an inversion is a pair $(a, b)$, such that $1 \leq a<b \leq n$ and $\pi(a)>\pi(b)$, and a non-inversion is a pair $(a, b)$, such that $1 \leq a<b \leq n$ and $\pi(a)<\pi(b)$. Denote the set of inversions of $\pi$ by $\operatorname{INV}(\pi)$, and the set of non-inversions by $\operatorname{NINV}(\pi)$.

Definition 2.3. Let $\pi$ be a permutation.

1. The number

$$
\operatorname{invsum}(\pi)=\sum_{(a, b) \in \operatorname{INV}(\pi)}(b-a),
$$

is called the inversion sum of $\pi$.

2. The number

$$
\operatorname{ninvsum}(\pi)=\sum_{(a, b) \in \operatorname{NINV}(\pi)}(b-a),
$$

is called the non-inversion sum of $\pi$.

Observe that the values added up in the sums are differences of positions $(b-a)$ rather than of values $(\pi(b)-\pi(a))$. The following result shows that had we defined the sums in terms of differences of values we would have resulted in the same function.

Proposition 2.4. For any permutation $\pi$

$$
\operatorname{ninvsum}\left(\pi^{\mathrm{i}}\right)=\operatorname{ninvsum}(\pi),
$$

or equivalently

$$
\sum_{(a, b) \in \operatorname{NINV}(\pi)}(\pi(b)-\pi(a))=\sum_{(a, b) \in \operatorname{NINV}(\pi)}(b-a) .
$$

A similar statement holds for the inversion sum.

Proof. We will prove the statement by induction on the rank of the permutation. Let $\pi$ be an arbitrary permutation and let $\pi(n)=k$. If $k=1$ then the result follows immediately by the induction hypotheses. Otherwise let $\pi\left(h_{j}\right)=j$ for $j=1, \ldots, k-1$. We depict in Figure 1 graphs of $\pi$ and $\pi^{\mathrm{i}}$, where the box ${ }_{i, j}$ represents the sets of pairs $\left(a, \pi_{a}\right)$ lying in the designated regions of the graph on the left, or $\left(a, \pi_{a}^{\mathrm{i}}\right)$ lying in the designated regions of the graph on the right. For example, box $_{2, j}=\left\{\left(a, \pi_{a}\right) \mid h_{j}<a, j<\pi_{a}<k\right\}$. Let $\tau$ be the permutation obtained from $\pi$ by removing the last element $k=\pi(n)$ and

\footnotetext{
${ }^{3}$ http://oeis.org/A135298
} 

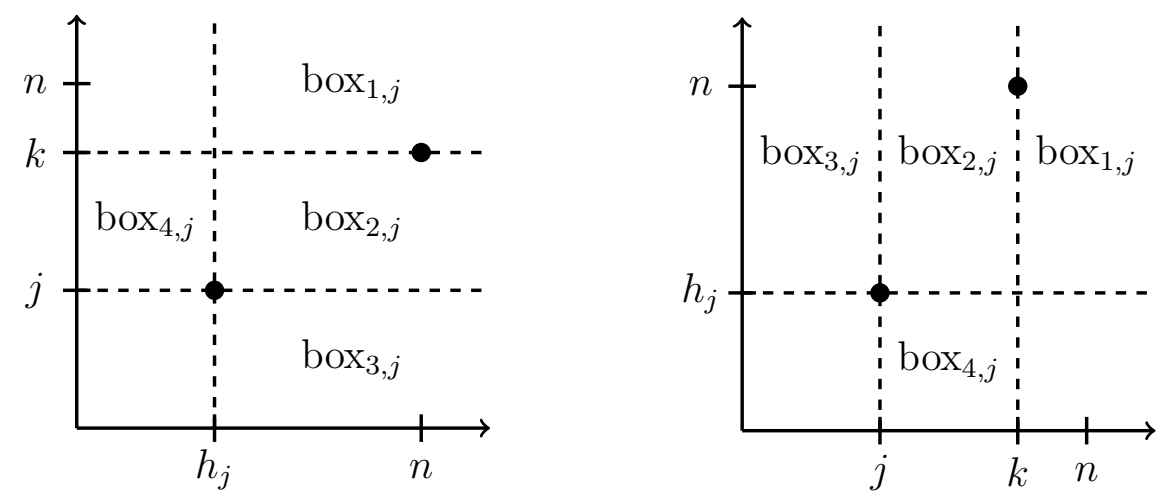

Figure 1: The permutation $\pi$ is shown on the left and $\pi^{\mathrm{i}}$ is shown on the right.

reducing the letters of $\pi$ that are larger than $k$ by 1 . Then, by the induction hypothesis, $\operatorname{ninvsum}(\tau)=\operatorname{ninvsum}\left(\tau^{\mathrm{i}}\right)$. But

$$
\operatorname{ninvsum}(\pi)=\operatorname{ninvsum}(\tau)+\sum_{j=1}^{k-1} 1+\left|\operatorname{box}_{1, j}\right|+\left|\operatorname{box}_{2, j}\right|+\left|\operatorname{box}_{3, j}\right|,
$$

where for each $j$ the sum of the box sizes is equal to one less than the separation $n-h_{j}$, and

$$
\operatorname{ninvsum}\left(\pi^{\mathrm{i}}\right)=\operatorname{ninvsum}\left(\tau^{\mathrm{i}}\right)+\sum_{j=1}^{k-1} 1+\left|\operatorname{box}_{1, j}\right|+\left|\operatorname{box}_{2, j}\right|+\left|\operatorname{box}_{4, j}\right|,
$$

where for each $j$ the sum of the box sizes box $_{2, j}$ and box $_{4, j}$ is equal to one less than the separation $k-j$ and the size of box $_{1, j}$ represents the number of former non-inversions whose separation has just increased by one.

To see that $\sum_{j=1}^{k-1}\left|\operatorname{box}_{3, j}\right|$ is equal to $\sum_{j=1}^{k-1}\left|\operatorname{box}_{4, j}\right|$ note that the following are equivalent:

- $(a, \sigma(a)) \in \operatorname{box}_{4, \pi(b)}$,

- $(a, b) \in \operatorname{INV}(\pi)$ with $\pi(a)<k$,

- $(b, \sigma(b)) \in \operatorname{box}_{3, \pi(a)}$.

It is straightforward to see that $\operatorname{ninvsum}\left(\pi^{\mathrm{r}}\right)=\operatorname{invsum}(\pi)=\operatorname{ninvsum}\left(\pi^{\mathrm{c}}\right)$.

Note that for any permutation $\pi$ of rank $n$, the sum of the inversion sum and the non-inversion sum is the $(n-1)^{\text {th }}$ tetrahedral number $\left(\begin{array}{c}n+1 \\ 3\end{array}\right)$ :

$$
\begin{aligned}
\operatorname{invsum}(\pi)+\operatorname{ninvsum}(\pi) & =\sum_{\substack{1 \leq a<b \leq n \\
(n-1) n(n+1)}}(b-a) \\
& =\frac{\left(\begin{array}{c}
n+1 \\
3
\end{array}\right),}{6}
\end{aligned}
$$


so two permutations have the same inversion sum if and only if they have the same non-inversion sum.

We now show that the cosine of the permutation is closely related to the non-inversion sum of the permutation.

Theorem 2.5. For any permutation $\pi$,

$$
\mathbf{1} \cdot \pi=\mathbf{1} \cdot \mathbf{1}^{\mathrm{c}}+\operatorname{ninvsum}(\pi) .
$$

Proof. Let $\varphi$ be a function mapping a permutation $\pi$ of rank $n$ to a vector, whose $j^{\text {th }}$ coordinate is the number of times the $j^{\text {th }}$ position of $\pi$ is at the end of a non-inversion minus the number of times the $j^{\text {th }}$ position is at the beginning of a non-inversion, that is,

$$
\varphi(\pi)_{j}=\sum_{(i, j) \in \operatorname{NINV}(\pi)} 1-\sum_{(j, k) \in \operatorname{NINV}(\pi)} 1 .
$$

The $j^{\text {th }}$ coordinate of $\varphi(\pi)$ is then the coefficient of $j$ (treating $j$ as a variable) in the non-inversion sum formula, and hence the contribution of the $j^{\text {th }}$ position of $\pi$ to the non-inversion sum is $j$ times this number. Thus $\operatorname{ninvsum}(\pi)=\mathbf{1} \cdot \varphi(\pi)$.

We next see that the $j^{\text {th }}$ coordinate of $\varphi(\pi)$ is $\varphi(\pi)_{j}=\pi_{j}-\mathbf{1}_{j}^{\text {c }}$. The first coordinate is $\varphi(\pi)_{1}=\pi_{1}-n=\pi_{1}-\mathbf{1}_{1}^{\mathrm{c}}$, since in the formula for the non-inversion sum, $\pi_{1}$ will be subtracted once for every non-inversion, which is guaranteed by a value greater than $\pi_{1}$. For general $j \geq 1$, if $\pi_{j}-\pi_{j+1}>0$, then $\varphi\left(\pi_{j+1}\right)$ can be obtained from $\varphi\left(\pi_{j}\right)$ by subtracting the number of values between $\pi_{j+1}$ and $\pi_{j}$, as given each such value $\pi_{k}$, either $k<j$, in which case $(k, j)$ was counted positively toward $\varphi\left(\pi_{j}\right)$ but $(k, j+1)$ does not count toward $\varphi\left(\pi_{j+1}\right)$, or $j>j+1$, in which case $(j, k)$ did not count toward $\varphi\left(\pi_{j}\right)$, but $(j+1, k)$ counts negatively toward $\varphi\left(\pi_{j+1}\right)$. Thus we subtract $\pi_{j}-\pi_{j+1}-1$. If $\pi_{j}-\pi_{j+1}<0$, then to obtain $\varphi\left(\pi_{j}\right)$ we add 1 for every value between $\pi_{j+1}$ and $\pi_{j}$, and we add 2 in order to account for the non-inversion $(j, j+1)$. Thus we add $\pi_{j+1}-\pi_{j}-1+2$. Either way, we obtain the formula:

$$
\varphi(\pi)_{j+1}=\varphi(\pi)_{j}+\pi_{j+1}-\pi_{j}-1 .
$$

By induction, let us assume that $\varphi(\pi)_{j}=\pi_{j}-\mathbf{1}_{j}^{\mathrm{c}}$. Thus

$$
\varphi(\pi)_{j+1}=\pi_{j}-\mathbf{1}_{j}^{\mathrm{c}}+\pi_{j+1}-\pi_{j}-1=\pi_{j+1}-\mathbf{1}_{j}^{\mathrm{c}}-1=\pi_{j+1}-\mathbf{1}_{j+1}^{\mathrm{c}} .
$$

In conclusion:

$$
\operatorname{ninvsum}(\pi)=\mathbf{1} \cdot \varphi(\pi)=\mathbf{1} \cdot\left(\pi-\mathbf{1}^{\mathrm{c}}\right)=\mathbf{1} \cdot \pi-\mathbf{1} \cdot \mathbf{1}^{\mathrm{c}}
$$

whence our desired result of this theorem immediately follows.

Note that for $\mathbf{1} \in \mathfrak{S}_{n}, \mathbf{1} \cdot \mathbf{1}^{\mathrm{c}}=\left(\begin{array}{c}n+2 \\ 3\end{array}\right)$, so equation 1 implies that the equation in the theorem is equivalent to

$$
\mathbf{1} \cdot \pi=\left(\begin{array}{c}
n+2 \\
3
\end{array}\right)+\left(\begin{array}{c}
n+1 \\
3
\end{array}\right)-\operatorname{invsum}(\pi),
$$


which can be simplified to

$$
\mathbf{1} \cdot \pi=\frac{n(n+1)(2 n+1)}{6}-\operatorname{invsum}(\pi) .
$$

Corollary 2.6. Given two permutations $\pi, \rho \in \mathfrak{S}_{n}$,

$$
\operatorname{ninvsum}(\pi \circ \rho)=\pi \cdot \rho^{\mathrm{i}}-\mathbf{1} \cdot \mathbf{1}^{\mathrm{c}} \text {. }
$$

Proof. By a direct calculation,

$$
\operatorname{ninvsum}(\pi \circ \rho)=\mathbf{1} \cdot(\pi \circ \rho)-\mathbf{1} \cdot \mathbf{1}^{\mathrm{c}}=\pi \cdot \rho^{\mathrm{i}}-\mathbf{1} \cdot \mathbf{1}^{\mathrm{c}}
$$

Observe that since $\pi \cdot \rho=\rho \cdot \pi$, then $\operatorname{ninvsum}\left(\pi \circ \rho^{\mathrm{i}}\right)=\operatorname{ninvsum}\left(\rho \circ \pi^{\mathrm{i}}\right)$. Then taking $\rho=$ $\mathbf{1}$, we get $\operatorname{ninvsum}(\pi)=\operatorname{ninvsum}\left(\pi^{\mathrm{i}}\right)$. This serves as an alternative proof to Proposition 2.4.

Lemma 2.7. For $n \geq 6$,

$$
\left(\begin{array}{c}
n+1 \\
3
\end{array}\right)+\left(\begin{array}{l}
n \\
3
\end{array}\right) \geq\left(\begin{array}{c}
n+2 \\
3
\end{array}\right)-1
$$

Proof. A straightforward calculation shows that for $n \geq 7,\left(\begin{array}{c}n+1 \\ 3\end{array}\right)+\left(\begin{array}{c}n \\ 3\end{array}\right)>\left(\begin{array}{c}n+2 \\ 3\end{array}\right)$. For the case where $n=6$, note that $\left(\begin{array}{l}7 \\ 3\end{array}\right)+\left(\begin{array}{l}6 \\ 3\end{array}\right)=\left(\begin{array}{l}8 \\ 3\end{array}\right)-1$.

Lemma 2.8. For each value $0 \leq k \leq 10$, there exists a permutation $\pi \in \mathfrak{S}_{4}$, such that $\operatorname{ninvsum}(\pi)=k$.

Proof. Here is a permutation for each value of $k: 4321,3421,3412,4213,4123,2413$, 3214, 1423, 2143, 1243, 1234.

Lemma 2.9. For $n \geq 4$ and each $0 \leq k \leq\left(\begin{array}{c}n+1 \\ 3\end{array}\right)$, there is a permutation $\pi \in \mathfrak{S}_{n}$, such that $\operatorname{ninvsum}(\pi)=k$.

Proof. We show this by induction on $n$, where the base case $(n=4)$ is given by Lemma 2.8 . Assuming this holds for $n-1$ (with $n>4$ ), we consider permutations $\pi \in \mathfrak{S}_{n}$, with $\pi_{n}=1$. The last entry does not contribute anything to the non-inversion sum of the first $n-1$, which by the induction hypothesis ranges through all the integers in the interval from 0 through $\left(\begin{array}{l}n \\ 3\end{array}\right)$. Next, consider permutations $\pi \in \mathfrak{S}_{n}$, with $\pi_{1}=1$. This first entry is guaranteed to contribute $\left(\begin{array}{l}n \\ 2\end{array}\right)$ to the non-inversion sum, while the rest can be chosen to contribute any integer ranging from 0 through $\left(\begin{array}{l}n \\ 3\end{array}\right)$. Because $\left(\begin{array}{c}n+1 \\ 3\end{array}\right)=\left(\begin{array}{l}n \\ 3\end{array}\right)+\left(\begin{array}{c}n \\ 2\end{array}\right)$, and because $\left(\begin{array}{l}n \\ 3\end{array}\right)>\left(\begin{array}{l}n \\ 2\end{array}\right)$ for $n>3$, we have that we can obtain every integer from 0 through $\left(\begin{array}{c}n+1 \\ 3\end{array}\right)$.

We are now ready to prove the main theorem of this section. 
Proof of Theorem 2.2. Given $k \geq 35$, let $n$ be the largest integer, such that $\left(\begin{array}{c}n+2 \\ 3\end{array}\right) \leq k$. Note that $n \geq 5$. Let $m=k-\left(\begin{array}{c}n+2 \\ 3\end{array}\right)$. For $n \geq 5$, we have by Lemma $2.7,\left(\begin{array}{c}n+2 \\ 3\end{array}\right)+\left(\begin{array}{c}n+1 \\ 3\end{array}\right) \geq$ $\left(\begin{array}{c}n+3 \\ 3\end{array}\right)-1$. Thus $m \leq\left(\begin{array}{c}n+1 \\ 3\end{array}\right)$, and hence by Lemma 2.9 , there is a permutation $\pi \in \mathfrak{S}_{n}$, with $\operatorname{ninvsum} \pi=m$. Thus, by Theorem 2.5 ,

$$
\mathbf{1} \cdot \pi=\left(\begin{array}{c}
n+2 \\
3
\end{array}\right)+\operatorname{ninvsum}(\pi)=\left(\begin{array}{c}
n+2 \\
3
\end{array}\right)+m=k
$$

For the values of $k$ less than 35, we first consider in the following chart for each $n \leq 5$, the maximum and minimum values $\mathbf{1} \cdot \pi$ can obtain, where $\pi \in \mathfrak{S}_{n}$.

\begin{tabular}{c|c|c}
$n$ & $\left(\begin{array}{c}n+2 \\
3\end{array}\right)$ & $\left(\begin{array}{c}n+2 \\
3\end{array}\right)+\left(\begin{array}{c}n+1 \\
3\end{array}\right)$ \\
\hline 1 & 1 & 1 \\
2 & 4 & 5 \\
3 & 10 & 14 \\
4 & 20 & 30 \\
5 & 35 & 55
\end{tabular}

By Lemma 2.9, we have permutations $\pi$ such that the value $\mathbf{1} \cdot \pi$ can hit every value from 20 through 30 . For the other values, we have the following chart

\begin{tabular}{c|c}
$\pi$ & $\mathbf{1} \cdot \boldsymbol{\pi}$ \\
\hline 1 & 1 \\
21 & 4 \\
12 & 5 \\
321 & 10 \\
312 & 11 \\
132 & 13 \\
123 & 14
\end{tabular}

Note that an integer $k \notin\{2,3,6,7,8,9,12,15,16,17,18,19,31,32,33,34\}$ is even if and only if there is a permutation $\pi$ such that $\mathbf{1} \cdot \pi=k$ and the number of odd integers in the odd positions of $\pi$ is even.

\subsection{Algorithm}

We present an algorithm for finding a permutation $\pi$ for a given $k \notin\{2,3,6,7,8,9,12,15$, $16,17,18,19,31,32,33,34\}$, such that $\mathbf{1} \cdot \pi=k$. We first introduce three functions: $\eta, r$, and $\nu$.

For $k<35$ and $k \notin\{2,3,6,7,8,9,12,15,16,17,18,19,31,32,33,34\}$, let $\eta(k)$ be $\pi$ such that $1 \cdot \pi=k$ (this is guaranteed by Lemma 2.2 and is easy to make explicit because of the bound on $k$ ).

Let $k$ be such that we wish to find $\pi$ with $\mathbf{1} \cdot \pi=k$. In the proof of Theorem 2.2 , we chose the length $n$ of the to-be-constructed $\pi$, such that $\left(\begin{array}{c}n+2 \\ 3\end{array}\right) \leq k$. Since $\left.6\left(\begin{array}{c}n+2 \\ 3\end{array}\right)-k\right)=$ 
$n^{3}+3 n^{2}+2 n-6 k$, we can determine from $k$ the desired $n$ as the floor of the real cubic root of $n^{3}+3 n^{2}+2 n-6 k$, which is the floor of

$$
\frac{1}{3} \sqrt[3]{81 k+3 \sqrt{(27 k)^{2}-3}}+\frac{1}{3} \sqrt[3]{81 k-3 \sqrt{(27 k)^{2}-3}}-1
$$

Let $r$ be a function mapping a positive integer $k$ to such a value $n$.

Let $\nu:\{0,1,2,3,4,5,6,7,8,9,10\} \rightarrow \mathfrak{S}_{4}$, be given by $0 \mapsto 4321,1 \mapsto 3421,2 \mapsto 3412$, $3 \mapsto 4213,4 \mapsto 4123,5 \mapsto 2413,6 \mapsto 3214,7 \mapsto 1423,8 \mapsto 2143,9 \mapsto 1243,10 \mapsto 1234$ This is from the proof of Lemma 2.8 .

Assuming the functions $\eta, r$, and $\nu$, we present an algorithm Main $(k)$, see Algorithm 1 , that calls another function $\zeta$, defined below in Algorithm 2, that inputs $m$, a value for the ninvsum, and $n$, the length of the permutation to create.
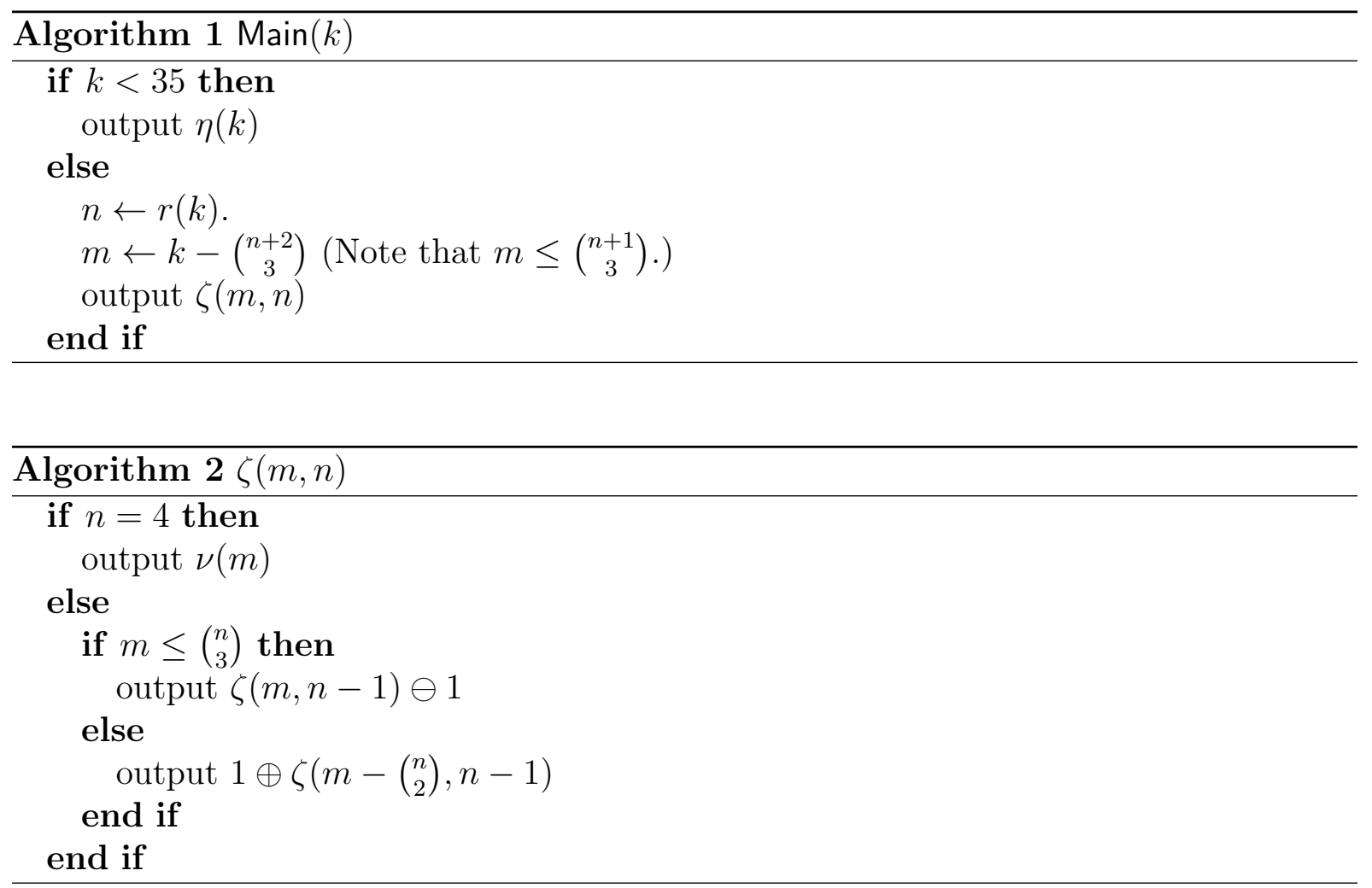

Here $\pi \oplus \sigma$ is the direct sum of the permutations $\pi$ and $\sigma$ and $\pi \ominus \sigma$ is the skew sum. Because of Lemma 2.7 and the fact that $n \geq 5$ for the first function call, we have that $m \leq\left(\begin{array}{c}n+1 \\ 3\end{array}\right)$ for that first call. The reasoning behind why the inductive hypothesis applies to Lemma 2.9 guarantees that $m \leq\left(\begin{array}{c}n+1 \\ 3\end{array}\right)$ for every function call after the first, even if $n=4$. Also, because of equation 2 , it is clear that the running time of this algorithm is proportional to $k^{1 / 3}$. 


\section{Zone-crossing vectors and the distribution of the non-inversion sum}

We are interested in the function

$$
N_{n}(x)=\sum_{\pi \in \mathfrak{S}_{n}} x^{\operatorname{ninvsum}(\pi)}
$$

which records the distribution of the non-inversion sum. Table 3 provides some empirical data generated with the computer algebra system Sage ${ }^{4}$, where we factor the polynomials as much as possible. In the context of Table 3, some of these polynomials factor into some reasonably small factors and a very large factor.

Table 1: The distribution function of ninvsum, $N_{n}(x)$.

\begin{tabular}{|c|c|c|}
\hline$n$ & Small factors of $N_{n}(x)$ & Big factor of $N_{n}(x)$ \\
\hline 1 & 1 & 1 \\
\hline 2 & $x+1$ & 1 \\
\hline 3 & 1 & $x^{4}+2 x^{3}+2 x+1$ \\
\hline 4 & $x^{2}+1$ & $x^{8}+3 x^{7}+x^{5}+2 x^{4}+x^{3}+3 x+1$ \\
\hline 5 & $x^{2}-x+1$ & $\begin{array}{l}x^{18}+5 x^{17}+7 x^{16}+8 x^{15}+8 x^{14}+6 x^{13}+2 x^{12}+6 x^{11}+10 x^{10} \\
+14 x^{9}+10 x^{8}+6 x^{7}+2 x^{6}+6 x^{5}+8 x^{4}+8 x^{3}+7 x^{2}+5 x+1\end{array}$ \\
\hline 6 & $(x+1)\left(x^{2}-x+1\right)^{2}$ & $\begin{array}{l}x^{30}+6 x^{29}+11 x^{28}+13 x^{27}+13 x^{26}+6 x^{25}-x^{24}+6 x^{23}+21 x^{22} \\
+30 x^{21}+19 x^{20}+3 x^{19}-7 x^{18}+14 x^{17}+27 x^{16}+36 x^{15}+27 x^{14} \\
14 x^{13}-7 x^{12}+3 x^{11}+19 x^{10}+30 x^{9}+21 x^{8}+6 x^{7}-x^{6}+6 x^{5} \\
+13 x^{4}+13 x^{3}+11 x^{2}+6 x+1\end{array}$ \\
\hline 7 & $\left(x^{2}-x+1\right)$ & $\begin{array}{l}x^{54}+7 x^{53}+16 x^{52}+23 x^{51}+36 x^{50}+39 x^{49}+38 x^{48}+45 x^{47}+62 x^{46} \\
+71 x^{45}+83 x^{44}+82 x^{43}+83 x^{42}+91 x^{41}+86 x^{40}+85 x^{39}+128 x^{38} \\
+149 x^{37}+144 x^{36}+129 x^{35}+132 x^{34}+101 x^{33}+137 x^{32}+166 x^{31} \\
+204 x^{30}+182 x^{29}+146 x^{28}+108 x^{27}+146 x^{26}+182 x^{25}+204 x^{24} \\
+166 x^{23}+137 x^{22}+101 x^{21}+132 x^{20}+129 x^{19}+144 x^{18}+149 x^{17} \\
+128 x^{16}+85 x^{15}+86 x^{14}+91 x^{13}+83 x^{12}+82 x^{11}+83 x^{10}+71 x^{9} \\
+62 x^{8}+45 x^{7}+38 x^{6}+39 x^{5}+36 x^{4}+23 x^{3}+16 x^{2}+7 x+1\end{array}$ \\
\hline 8 & $\left(x^{4}+1\right)\left(x^{2}-x+1\right)$ & $\begin{array}{l}x^{78}+8 x^{77}+22 x^{76}+36 x^{75}+60 x^{74}+71 x^{73}+66 x^{72}+67 x^{71}+84 x^{70} \\
+94 x^{69}+133 x^{68}+150 x^{67}+171 x^{66}+182 x^{65}+164 x^{64}+135 x^{63} \\
+196 x^{62}+249 x^{61}+280 x^{60}+278 x^{59}+290 x^{58}+218 x^{57}+243 x^{56} \\
+270 x^{55}+375 x^{54}+456 x^{53}+432 x^{52}+326 x^{51}+322 x^{50}+329 x^{49} \\
+442 x^{48}+481 x^{47}+533 x^{46}+464 x^{45}+413 x^{44}+362 x^{43}+437 x^{42} \\
+489 x^{41}+520 x^{40}+462 x^{39}+520 x^{38}+489 x^{37}+437 x^{36}+362 x^{35} \\
+413 x^{34}+464 x^{33}+533 x^{32}+481 x^{31}+442 x^{30}+329 x^{29}+322 x^{28} \\
+326 x^{27}+432 x^{26}+456 x^{25}+375 x^{24}+270 x^{23}+243 x^{22}+218 x^{21} \\
+290 x^{20}+278 x^{19}+280 x^{18}+249 x^{17}+196 x^{16}+135 x^{15}+164 x^{14} \\
+182 x^{13}+171 x^{12}+150 x^{11}+133 x^{10}+94 x^{9}+84 x^{8}+67 x^{7}+66 x^{6} \\
+71 x^{5}+60 x^{4}+36 x^{3}+22 x^{2}+8 x+1\end{array}$ \\
\hline
\end{tabular}

One can observe that the degree of $N_{n}(x)$ is always the $(n-1)^{\text {th }}$ tetrahedral number $\left(\begin{array}{c}n+1 \\ 3\end{array}\right)$. This is consistent with equation $(1)$, where the maximum non-inversion sum $\left(\begin{array}{c}n+1 \\ 3\end{array}\right)$ can be obtained using the identity permutation 1 .

The primary aim of this section is to find a recursive definition of the distribution function for the non-inversion sum, that is, to define $N_{n+1}(x)$ in terms of $N_{n}(x)$. Our formulation of the distribution function will involve a new type of vector, the zone-crossing vector, whose coordinates count the number of inversions or non-inversions $(a, b)$ of a permutation, with a given point between $a$ and $b$.

\footnotetext{
${ }^{4}$ www.sagemath.org
} 
Definition 3.1. Given a permutation $\pi$ of rank $n$, we define

1. its inversion zone-crossing vector, $\operatorname{izcv}(\pi)=\left(z_{1}, z_{2}, \ldots, z_{n-1}\right)$, where $z_{k}$ is the number of inversions $(a, b) \in \operatorname{INV}(\pi)$, where $a \leq k<b$, and its augmented zone crossing vector $\operatorname{aizcv}(\pi)=\left(0, z_{1}, z_{2}, \ldots, z_{n-1}, 0\right)$.

2. its non-inversion zone-crossing vector $\operatorname{nzcv}(\pi)=\left(z_{1}, z_{2}, \ldots, z_{n-1}\right)$, where $z_{k}$ is the number of non-inversions $(a, b) \in \operatorname{NINV}(\pi)$, where $a \leq k<b$, and its augmented zone crossing vector $\operatorname{anzcv}(\pi)=\left(0, z_{1}, z_{2}, \ldots, z_{n-1}, 0\right)$.

Example 3.2. Consider the permutation $\pi=314562$. Then $\operatorname{izcv}(\pi)=(2,1,2,3,4)$ and $\operatorname{nzcv}(\pi)=(3,7,7,5,1)$.

The following proposition states that a zone crossing vector uniquely determines its permutation.

Proposition 3.3. If $v=\left(v_{0}, v_{1}, \ldots, v_{n-1}, v_{n}\right)=\operatorname{aizcv}(\pi)$, then $\pi_{k}=n-(k-1)-\left(v_{k}-\right.$ $\left.v_{k-1}\right)$, for $1 \leq k \leq n$. (Therefore, if $\rho$ is a permutation, such that $\operatorname{aizcv}(\pi)=\operatorname{aizcv}(\rho)$, then $\pi=\rho$.)

Proof. Let $\rho$ be a permutation, and let $v$ be its zone-crossing vector. Let $\pi$ be constructed according to the statement of the proposition. We show that $\pi=\rho$. First observe that $\rho_{1}=n-v_{1}$, since this is the number of positions to the right of the first position that have a value greater than $\rho_{1}$. Thus $\rho_{1}=\pi_{1}$. For a general $k \geq 1$, note that if $\rho_{k}=1$, then $v_{k}-v_{k-1}=n-k$. Thus $1=\rho_{k}=n-(k-1)-\left(v_{k}-v_{k-1}\right)$, just as is the case with $\pi_{k}$. To consider different values of $\rho_{k}$, imagine incrementing its value by 1 as a result of swapping $\rho_{k}$ with the position with one larger value. If $\rho_{k}$ is incremented by 1 , then $v_{k}-v_{k-1}$ is decremented by 1 , for either the original value of $\rho_{k}$ is swapped with a value to the right, thus decrementing $v_{k}$, or it is swapped with a value to the left, thus incrementing $v_{k-1}$. Thus all the values of $\rho_{k}$ can be obtained by the formula above, and hence $\rho=\pi$.

Lemma 3.4. The sum of the coordinates of $\operatorname{nzcv}(\pi)$ equals ninvsum $(\pi)$.

Proof. This follows from the fact that each non-inversion will contribute to as many zonecrossing coordinates as is the separation distance of the non-inversion. For example, a non-inversion from position 1 to position 3 has separation 2 , which is the number of zone-crossing coordinates it will contribute to.

Proposition 3.5. For any $\pi \in \mathfrak{S}_{n}$,

1. $\operatorname{nzcv}(\pi)+\operatorname{izcv}(\pi)=(1 \cdot n-1,2 \cdot n-2, \ldots, n-1 \cdot 1)$,

2. $\operatorname{nzcv}\left(\pi^{\mathrm{c}}\right)=\operatorname{izcv}(\pi)$,

3. $\operatorname{nzcv}\left(\pi^{\mathrm{r}}\right)=\operatorname{izcv}(\pi)$. 
Proof. 1. To prove $\operatorname{nzcv}(\pi)+\operatorname{izcv}(\pi)=(1 \cdot n-1,2 \cdot n-2, \ldots, n-1 \cdot 1)$, we note that the $j^{\text {th }}$ coordinate of $\operatorname{nzcv}(\pi)+\operatorname{izcv}(\pi)$ counts the total number of pairs matching each coordinate in the first $j$ positions with each coordinate in the last $n-j$ positions. This is because each such pair is a zone crossing inversion or non-inversion and is hence counted in either $\operatorname{izcv}(\pi)$ or $\operatorname{nzcv}(\pi)$. This yields the vector $(1 \cdot n-1,2 \cdot n-$ $2, \ldots, n-1 \cdot 1)$, giving us the desired formula.

2. To prove $\operatorname{nzcv}\left(\pi^{\mathrm{c}}\right)=\operatorname{izcv}(\pi)$, note that the complement operation changes every inversion to a non-inversion, and every non-inversion to an inversion.

3. To prove $\operatorname{nzcv}\left(\pi^{\mathrm{r}}\right)=\operatorname{izcv}^{\mathrm{r}}(\pi)$, note that every inversion in $\pi$ between positions $j$ and $j+k$ is a non-inversion in $\pi^{\mathrm{r}}$ from positions $n-j-k+1$ to $n-j+1$. This yields $\operatorname{nzcv}^{\mathrm{r}}\left(\pi^{\mathrm{r}}\right)=\mathrm{izcv}$, and reversing the vectors on each side yields the desired equation.

Lemma 3.6. Let $\pi$ be a permutation of rank $n$ with augmented zone-crossing vector $\operatorname{anzcv}(\pi)=\left(a_{0}, a_{1}, a_{2}, \ldots, a_{n-1}, a_{n}\right)$. Let $\rho$ be the permutation obtained by inserting $n+1$ into $\pi$ in between position $k$ and $k+1$ (in the case where $k=0$, the resulting permutation is $1 \ominus \pi)$. Then for $0 \leq k \leq n$,

$$
\operatorname{anzcv}(\rho)=\left(a_{0}+0, a_{1}+1, a_{2}+2, \ldots,\left(a_{k}+k\right), a_{k}, a_{k+1}, \ldots, a_{n}\right) .
$$

(Note that for $k=0$, we have $\operatorname{anzcv}(\rho)=\left(0,0, a_{1}, a_{2}, \ldots, a_{n-1}, 0\right)$ and for $k=n$ we have $\operatorname{anzcv}(\rho)=\left(0, a_{1}+1, a_{2}+2, \ldots, a_{n-1}+n-1, n, 0\right)$. $)$

Proof. For $0 \leq j \leq k$ the $j^{\text {th }}$ position of the zone-crossing vector (counting from 0 in the augmented vector) is incremented by the number $j$ of positions in the left zone, as each forms a new non-inversion pair with the new position $k+1$ in the right zone. The $j^{\text {th }}$ position among the $(k+1)^{\text {th }}$ position in the new zone-crossing vector counts the number of non-inversions starting among the first $j$ positions and ending among the $n+1-j$ positions. As the inserted position is now in the left zone and has the highest value, it does not contribute to the zone-crossing count. Thus the $j^{\text {th }}$ position of the new zone-crossing vector is the same as the $(j-1)^{\text {th }}$ position of the old zone-crossing vector (we decrement the position by one, as the position counts the size of the left zone, which decreases by one when the inserted position is removed).

The preceding lemma can be used to give a recursive definition of the zone-crossing vectors. We show how this is done when adding a value to permutations of rank 2 to obtain permutations of rank 3. The set of (augmented) non-inversion zone-crossing vectors for permutations of rank 2 are (010) corresponding to the permutation 12 and (000) corresponding to 21. Using Lemma 3.6, we determine the (augmented) non-inversion zone crossing vectors for permutations of rank 3 in the following chart:

\begin{tabular}{c|c|c|c} 
& $k=0$ & $k=1$ & $k=2$ \\
\hline$(000)$ & $(0 \underline{000)}$ & $(01 \underline{00})$ & $(012 \underline{0})$ \\
$(010)$ & $(0 \underline{0} 10)$ & $(02 \underline{1} 0)$ & $(022 \underline{0})$
\end{tabular}


The first column gives the zone-crossing vectors for the permutations of rank 2 . The first row gives the position $k$ to the right of which we place the highest value to obtain the new permutation. The remaining entries correspond to the resulting zone-crossing vectors. We underline the value $a_{k}$ in position $k+1$ of the zone-crossing vector. Notice that these correspond to the positions $k$ in the left column.

In summary, we see that these vectors do not range across all possibilities between the lowest (0000) and the highest (0220), as we are missing (0110), (0020) and (0200). We hope future work can yield a more direct characterization of the set of all possible zone-crossing vectors. Such a characterization may reveal new patterns of a variety of permutation statistics. A variation of such a characterization might, for example, capture sets of zone-crossing vectors correspond to permutations avoiding a certain classical pattern of rank 3, such as the patten 231. Since the number of permutations of rank $n$ that avoid a given classical pattern of rank 3 is the $n^{\text {th }}$ Catalan number, such a set may offer a new Catalan structure.

The proof of the following theorem will make use of partitions of integers. Given a positive integer, $n$, we write $\lambda \dashv n$ to indicate that $\lambda$ is a partition of $n$. We write $\ell_{\lambda}$ for the length of the partition $\lambda$. For example, $\lambda=3+3+2+1$ is a partition of 9 of length 4 .

Theorem 3.7. The number of permutations of rank $n$ such that the $k^{\text {th }}$ coordinate of the zone-crossing vector equals $\ell$ is

$$
k !(n-k) !\left[q^{\ell}\right]\left[\begin{array}{l}
n \\
k
\end{array}\right]_{q} .
$$

In other words

$$
\sum_{\pi \in \mathfrak{S}_{n}} q^{\mathrm{nzcv}(\pi)_{k}}=k !(n-k) !\left[\begin{array}{l}
n \\
k
\end{array}\right]_{q} .
$$

Proof. The number of partitions of $\ell$ into at most $k$ parts, where each part has size at most $n-k$, is given by $\left[q^{\ell}\right]\left[\begin{array}{l}n \\ k\end{array}\right]_{q}$. Each part may correspond to one of the first $k$ positions of a permutation, and the size of the part would correspond to the number out of the $n-k$ last positions that the selected position is a non-inversion with. For each partition, we may rearrange the first $k$ positions and rearrange the last $n-k$ positions without affecting the $k^{\text {th }}$ coordinate of the zone-crossing vector. This gives us the remaining $k !(n-k)$ !.

Theorem 3.8. For $n \geq 1$, letting $\left(\begin{array}{l}1 \\ 2\end{array}\right)=0$, we have

$$
\begin{aligned}
N_{n+1}(q) & =\sum_{k=0}^{n} q^{\left(\begin{array}{c}
k+1 \\
2
\end{array}\right)} \sum_{\pi \in \mathfrak{S}_{n}} q^{\operatorname{anzcv}_{k}(\pi)} q^{\text {ninvsum }(\pi)} \\
& =N_{n}(q)+\sum_{k=1}^{n-1} q^{\left(\begin{array}{c}
k+1 \\
2
\end{array}\right)} \sum_{\pi \in \mathfrak{S}_{n}} q^{\mathrm{nzcv}_{k}(\pi)} q^{\text {ninvsum }(\pi)}+q^{\left(\begin{array}{c}
n+1 \\
2
\end{array}\right)} N_{n}(q) .
\end{aligned}
$$

THE EleCtronic JOURNAL OF COMBINATORICS 19 (2012), \#P29 
Proof. We are interested in the result of extending a permutation $\pi$ to a permutation $\rho$ by inserting $n+1$ between the $k^{\text {th }}$ and $(k+1)^{\text {th }}$ positions of $\pi$ (like in Lemma 3.6). We proceed by summing the functions restricted to permutations with $n+1$ in the $(k+1)^{\text {th }}$ positions for each $k$. When $k=0$, the value $n+1$ is inserted at the beginning of the permutation and hence contributes nothing to the non-inversion sum. Thus we have the term $N_{n}(q)$. When $k=n$, the value $n+1$ is in the last position, and hence adds (the maximum) $\left(\begin{array}{c}n+1 \\ 2\end{array}\right)$ to whatever non-inversion sum the permutation originally had. Thus our last term will be $q^{\left(\begin{array}{c}n+1 \\ 2\end{array}\right)} N_{n}(q)$. For the other values of $k(1 \leq k \leq n-1)$, what the insertion of the value $n+1$ contributes to the non-inversion sum depends on the original permutation. By Lemma 3.6, the sum of the zone-crossing vector coordinates (equaling the non-inversion sum) increases by $\left(\begin{array}{c}k+1 \\ 2\end{array}\right)+\mathrm{nzcv}_{k}$, which is why we multiply $q^{\text {ninvsum }(\pi)}$ by $q^{\left(\begin{array}{c}k+1 \\ 2\end{array}\right)+\mathrm{nzcv}_{k}(\pi)}$.

\section{The distribution of $k-,\left(k_{1}, k_{2}\right)$-, and $(\leq k)$-step in- versions}

\subsection{The distribution of $k$-step inversions}

Definition 4.1. A $k$-step inversion of a permutation $\pi$ is an inversion $(a, b) \in \operatorname{INV}(\pi)$, where $b-a=k$. Similarly a $k$-step non-inversion is a non-inversion $(a, b) \in \operatorname{NINV}(\pi)$, such that $b-a=k$.

Let $\operatorname{inv}_{k}(\pi)$ be the number of $k$-step inversions in $\pi$, and let $\operatorname{ninv}_{k}(\pi)$ be the number of $k$-step non-inversions in $\pi$. Then $\operatorname{inv}(\pi)=\sum_{k=1}^{n-1} \operatorname{inv}_{k}(\pi)$, and similarly for $\operatorname{ninv}_{k}(\pi)$. Define

$$
H_{n, k}(x)=\sum_{\pi \in \mathfrak{S}_{n}} x^{\mathrm{inv}_{k}(\pi)}
$$

so $I(n, k, i) \stackrel{\text { def }}{=}\left[x^{i}\right] H_{n, k}(x)$ is the number of permutations in $\mathfrak{S}_{n}$ with the number of $k$-step inversions equaling the number $i$. It is known that $H_{n, 1}(x)$ is the $n^{\text {th }}$ Eulerian polynomial, which we denote $A_{n}(x)^{5}$, since a 1-step inversion is a descent.

In finding $H_{n, k}(x)$ for arbitrary $k$, we will divide up the permutations into $k$ smaller permutations which can be interleaved to form the original. We call these smaller permutations runs, and define them precisely as follows.

Definition 4.2. Given a permutation $\pi$ of rank $n$ and $1 \leq k \leq n$, the $i^{\text {th }} k$-step run of $\pi$ is the permutation $\rho$ of $\operatorname{rank} j=\lfloor(n-i) / k\rfloor+1$, where $\rho_{j}=\pi_{k(j-1)+i}$.

Let $\lambda_{i}=\lfloor(n-i) / k\rfloor+1$ be the length of the $i^{\text {th }} k$-step permutation. One can observe that if $n \geq k$, then there are $\operatorname{rem}(n / k)$ many $j$, such that $\lambda_{j}=\lfloor n / k\rfloor+1$, and $k-\operatorname{rem}(n / k)$ many $j$, such that $\lambda_{j}=\lfloor n / k\rfloor$. Furthermore, $\sum_{i=1}^{k} \lambda_{i}=n$. The intuition for this can be seen in the following example, where the runs partition the original permutation, and hence the $\lambda_{i}$ partition $n$.

\footnotetext{
${ }^{5}$ The coefficient $\left[x^{k-1}\right] A_{n}(x)$ is $T(n, k)$ in the sequence http://oeis.org/A008292
} 
Example 4.3. Consider the case $n=11, k=4$. Since $n \geq k$, the total number of 4 -step runs is four. Of those, three are of length $3\left(\lambda_{1}, \lambda_{2}, \lambda_{3}=3\right)$.

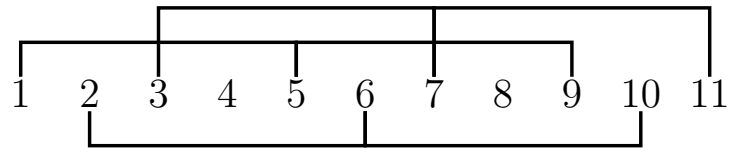

The remaining one is of length $2\left(\lambda_{4}=2\right)$.

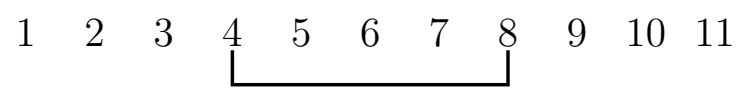

Note that $k$-step inversions only occur within the same $k$-step run, and that a $k$-step inversion in the original permutation corresponds to a 1-step inversion (a descent) in a run. We will see in the proof of the next theorem how this leads to $H_{11,4}=I(11,4,0) A_{3}^{3}(x) A_{2}^{1}(x)$, where $I(11,4,0)$ will count the ways of distributing the 11 values among the 4 runs, and the $A_{s}$ will correspond to the permutations of the $s$ values within a run.

Theorem 4.4. For $1 \leq k \leq n$ let $s=\lfloor n / k\rfloor+1$ and $t=\operatorname{rem}(n / k)$. Then

$$
H_{n, k}(x)=I(n, k, 0) A_{s}^{t}(x) A_{s-1}^{k-t}(x),
$$

where $A_{\ell}(x)=H_{\ell, 1}(x)$ is the $\ell^{\text {th }}$ Eulerian polynomial, and

$$
I(n, k, 0)=\prod_{j=1}^{k-1}\left(\begin{array}{c}
n-\sum_{i=0}^{j-1} \lambda_{i} \\
\lambda_{j}
\end{array}\right)
$$

where the $\lambda_{0} \stackrel{\text { def }}{=} 0$ and for $1 \leq j \leq k-1, \lambda_{j}$ is the length of the $j^{\text {th }} k$-step run.

Proof. Let us first find $I(n, k, 0)$. Given a permutation $\pi$ with no $k$-step inversions, the order within each run must be increasing. Thus the entire variation of such permutations is with how the $n$ values are distributed among the runs. If $j-1$ runs have been filled, we must choose $\lambda_{j}$ more out of the remaining $n-\sum_{i=0}^{j-1} \lambda_{i}$. Thus we have

$$
I(n, k, 0)=\prod_{j=1}^{k-1}\left(\begin{array}{c}
n-\sum_{i=0}^{j-1} \lambda_{i} \\
\lambda_{j}
\end{array}\right) .
$$

Furthermore, the number of $k$-step inversions is invariant over how we distribute $n$ among the runs. Thus $I(n, k, 0)$ is a factor of the distribution function. What the number of $k$-step inversions depends on is how the numbers are arranged within each run. Note again that $k$-step inversions only occur within the same $k$-step run, and that a $k$-step inversion in the original permutation corresponds to a 1-step inversion (a descent) in a run. In this way, the runs do not interact. Thus

$$
I(n, k, i)=\sum_{\sum_{j=1}^{k} p_{j}=i ; 1 \leq p_{j}<\lambda_{j}} I(n, k, i) \prod_{j=1}^{k}\left[x^{p_{j}}\right] A_{\lambda_{j}}(x) .
$$


Since there are $t=\operatorname{rem}(n / k)$ many $j$, such that $\lambda_{j}=s=\lfloor n / k\rfloor+1$, and $k-\operatorname{rem}(n / k)$ many $j$, such that $\lambda_{j}=\lfloor n / k\rfloor$, we have that

$$
H_{n, k}(x)=I(n, k, 0) A_{s}^{t}(x) A_{s-1}^{k-t}(x) .
$$

If we had used $\operatorname{ninv}_{k}$ instead of inv $v_{k}$ in the definition of the distribution function $H_{n, k}(x)$ then we would have obtained the same formula as in the theorem above. Also, had we used the difference of values, rather than positions, in the definitions of inv $k_{k}$ and ninv $_{k}$, we would also have arrived at the same formula, since the values-definition for $\pi$ would have corresponded to the positions-definition for $\pi^{\mathrm{i}}$. Table 2 includes experimental runs for the distribution function $H_{n, k}$ for $n=1, \ldots, 9$, and select $k$ for high values of $n$.

Table 2: The distribution function of $\operatorname{ninv}_{k}, H_{n, k}(x)$.

\begin{tabular}{|c|c|c|}
\hline$n$ & $k$ & $H_{n, k}(x)$ \\
\hline 1 & 1 & 1 \\
\hline 2 & $\begin{array}{l}1 \\
2\end{array}$ & $\begin{array}{l}x+1 \\
2\end{array}$ \\
\hline 3 & $\begin{array}{l}1 \\
2 \\
3\end{array}$ & $\begin{array}{l}x^{2}+4 x+1 \\
3(x+1) \\
6\end{array}$ \\
\hline 4 & $\begin{array}{l}1 \\
2 \\
3 \\
4\end{array}$ & $\begin{array}{l}(x+1)\left(x^{2}+10 x+1\right) \\
6(x+1)^{2} \\
12(x+1) \\
24\end{array}$ \\
\hline 5 & $\begin{array}{l}1 \\
2 \\
3 \\
4 \\
5\end{array}$ & $\begin{array}{l}x^{4}+26 x^{3}+66 x^{2}+26 x+1 \\
10(x+1)\left(x^{2}+4 x+1\right) \\
30(x+1)^{2} \\
60(x+1) \\
120\end{array}$ \\
\hline 6 & $\begin{array}{l}1 \\
2 \\
3 \\
4 \\
5 \\
6\end{array}$ & $\begin{array}{l}(x+1)\left(x^{4}+56 x^{3}+246 x^{2}+56 x+1\right) \\
20\left(x^{2}+4 x+1\right)^{2} \\
90(x+1)^{3} \\
180(x+1)^{2} \\
360(x+1) \\
720\end{array}$ \\
\hline 7 & $\begin{array}{l}1 \\
2 \\
3 \\
4 \\
5 \\
6 \\
7\end{array}$ & $\begin{array}{l}x^{6}+120 x^{5}+1191 x^{4}+2416 x^{3}+1191 x^{2}+120 x+1 \\
35(x+1)\left(x^{2}+4 x+1\right)\left(x^{2}+10 x+1\right) \\
210(x+1)^{2}\left(x^{2}+4 x+1\right) \\
630(x+1)^{3} \\
1260(x+1)^{2} \\
2520(x+1) \\
5040\end{array}$ \\
\hline 8 & $\begin{array}{l}1 \\
2 \\
3 \\
4 \\
5 \\
6 \\
7 \\
8\end{array}$ & $\begin{array}{l}(x+1)\left(x^{6}+246 x^{5}+4047 x^{4}+11572 x^{3}+4047 x^{2}+246 x+1\right) \\
70(x+1)^{2}\left(x^{2}+10 x+1\right)^{2} \\
560(x+1)\left(x^{2}+4 x+1\right)^{2} \\
2520(x+1)^{4} \\
5040(x+1)^{3} \\
10080(x+1)^{2} \\
20160(x+1) \\
40320\end{array}$ \\
\hline 9 & $\begin{array}{l}1 \\
2 \\
3 \\
4\end{array}$ & $\begin{array}{l}x^{8}+502 x^{7}+14608 x^{6}+88234 x^{5}+156190 x^{4}+88234 x^{3}+14608 x^{2}+502 x+1 \\
126(x+1)\left(x^{2}+10 x+1\right)\left(x^{4}+26 x^{3}+66 x^{2}+26 x+1\right) \\
1680\left(x^{2}+4 x+1\right)^{3} \\
7560(x+1)^{3}\left(x^{2}+4 x+1\right) \\
\end{array}$ \\
\hline
\end{tabular}

\section{$4.2\left(k_{1}, k_{2}\right)$-step inversions and non-inversions}

Definition 4.5. Given a permutation $\pi$, a $\left(k_{1}, k_{2}\right)$-step inversion is a pair $(a, b)$, such that $1 \leq a<b \leq n$, satisfying $b-a=k_{1}$ and $\pi(b)-\pi(a)=k_{2}$. Similarly, a $\left(k_{1}, k_{2}\right)$ - 
step non-inversion is a pair $(a, b)$, such that $1 \leq a<b \leq n$, satisfying $b-a=k_{1}$ and $\pi(a)-\pi(b)=k_{2}$.

Let $\operatorname{inv}_{\left(k_{1}, k_{2}\right)}(\pi)$ be the number of $\left(k_{1}, k_{2}\right)$-step inversions in $\pi$. Then

$$
\operatorname{inv}(\pi)=\sum_{1 \leq k_{1}, k_{2} \leq n-1} \operatorname{inv}_{\left(k_{1}, k_{2}\right)}(\pi)
$$

Define

$$
H_{n,\left(k_{1}, k_{2}\right)}(x)=\sum_{\pi \in \mathfrak{S}_{n}} x^{\mathrm{inv}_{\left(k_{1}, k_{2}\right)}(\pi)}
$$

Proposition 4.6. Let $n / 2<k_{1}, k_{2}<n$. Then the degree of $H_{n,\left(k_{1}, k_{2}\right)}(x)$ is $\ell=\min (n-$ $\left.k_{1}, n-k_{2}\right)$, and its leading coefficient equals

$$
(n-2 \ell) ! \ell !\left(\begin{array}{c}
n-k_{1} \\
\ell
\end{array}\right)\left(\begin{array}{c}
n-k_{2} \\
\ell
\end{array}\right) .
$$

Proof. Since $k_{1}>n / 2$, there are $n-k_{1}$ location pairs that a $k_{1}$-step inversion could be, since there are $n-k_{1}$ many $k$-step runs of length two, with the remainder of the runs of length 1 . Similarly, since $k_{2}>n / 2$, there are at most $n-k_{2}$-inversions with a value separation of $k_{2}$, as the top value has to be greater than $k_{2}$. Thus $\ell=\min \left(n-k_{1}, n-k_{2}\right)$ is the maximum number of $\left(k_{1}, k_{2}\right)$-step inversions, and the degree of $H_{n,\left(k_{1}, k_{2}\right)}(x)$ is $\ell$.

For the leading coefficient, we select $\left(\begin{array}{c}n-k_{1} \\ \ell\end{array}\right)$ positions pairs to place top values among $\left(\begin{array}{c}n-k_{2} \\ \ell\end{array}\right)$. Then there are $\ell$ ! ways to arrange the values among the position pairs, and there are $(n-2 \ell)$ ! ways to arrange the remaining values among the remaining positions. Note that no new $\left(k_{1}, k_{2}\right)$-step inversions can occur with the $(n-2 \ell)$ ! values and positions, since either all the available position pairs have been filled (when $\ell=n-k_{1}$ ) or all the available top values have been used (when $\ell=n-k_{2}$ ).

This proof will be adapted in Section 5.1, for a similar result involving $k$-step inversions with inversion tops divisible by $d$. For future work, we would like a complete description of the polynomials $H_{n,\left(k_{1}, k_{2}\right)}(x)$ as we had for $H_{n, k}(x)$, the distribution of $k$-step inversions. Table 3 contains some experimental runs for $H_{n,\left(k_{1}, k_{2}\right)}(x)$.

\subsection{The distribution of $(\leq k)$-step inversions}

Definition 4.7. Let

$$
\operatorname{inv}_{\leq k}(\pi)=\sum_{k^{\prime} \leq k} \operatorname{inv}_{k^{\prime}}(\pi)
$$

Then, since $n-1$ is the maximum separation, and a separation of one corresponds to a descent, we get

$$
\operatorname{inv}(\pi)=\operatorname{inv}_{\leq n-1}(\pi) \quad \text { and } \quad \operatorname{des}(\pi)=\operatorname{inv}_{\leq 1}(\pi)
$$


Table 3: The distribution function of $\operatorname{inv}_{\left(k_{1}, k_{2}\right)}, H_{n,\left(k_{1}, k_{2}\right)}(x)$.

\begin{tabular}{|l|l|l|l|l|l|}
\hline$n$ & $k_{1}$ & $k_{2}=1$ & $k_{2}=2$ & $k_{2}=3$ & $k_{2}=4$ \\
\hline 1 & 1 & 1 & & & \\
\hline 2 & 1 & $x+1$ & 2 & & \\
& 2 & 2 & 2 & & \\
\hline 3 & 1 & $x^{2}+2 x+3$ & $2(x+2)$ & 6 & \\
& 2 & $2(x+2)$ & 6 & 6 & \\
& 3 & 6 & $2\left(x^{2}+4 x+7\right)$ & 6 & \\
\hline 4 & 1 & $x^{3}+3 x^{2}+9 x+11$ & $2\left(x^{2}+2 x+9\right)$ & $4(x+3)$ & 24 \\
& 2 & $2\left(x^{2}+4 x+7\right)$ & $4(x+5)$ & $2(x+11)$ & 24 \\
& 3 & $6(x+3)$ & 24 & 24 & 24 \\
& 4 & 24 & $2\left(x^{3}+6 x^{2}+21 x+32\right)$ & $6\left(x^{2}+6 x+13\right)$ & $24(x+4)$ \\
\hline 5 & 1 & $x^{4}+4 x^{3}+18 x^{2}+44 x+53$ & $(x+2)$ & $6(3 x+17)$ \\
& 2 & $2\left(x^{3}+6 x^{2}+21 x+32\right)$ & $(x+3)\left(x^{2}+4 x+25\right)$ & $4\left(x^{2}+7 x+22\right)$ & $2(x+2)$ \\
& 3 & $6\left(x^{2}+6 x+13\right)$ & $4\left(x^{2}+7 x+22\right)$ & $2\left(x^{2}+10 x+49\right)$ & $12(x+9)$ \\
& 4 & $24(x+4)$ & $6(3 x+17)$ & $12(x+9)$ & $6(x+19)$ \\
& 5 & 120 & 120 & 120 & 120 \\
\hline
\end{tabular}

for any permutation of rank $n$. Define

$$
J_{n, \leq k}(x)=\sum_{\pi \in \mathfrak{S}_{n}} x^{\mathrm{inv} \leq k(\pi)}
$$

For the purpose of the next proposition we recall the falling factorial

$$
(k)_{j}=k(k-1) \cdots(k-(j-1)),
$$

and define a differential operator

$$
\nabla_{k}=\sum_{j=0}^{k} \frac{j+1}{(k)_{j}} \frac{\mathrm{d}^{j}}{\mathrm{~d} x^{j}}
$$

Proposition 4.8. 1. If the maximum step-size is 1, we have

$$
J_{n, 1}(x)=A_{n}(x)
$$

where $A_{n}(x)$ is the $n^{\text {th }}$ Eulerian polynomial.

2. If the maximum step-size is $n-2$, we have

$$
J_{n, \leq n-2}(x)=J_{n-1, \leq n-3}(x) \cdot\left(\frac{\mathrm{d}}{\mathrm{d} x}\left(x \sum_{j=0}^{n-2} x^{j}\right)+x^{n-2} \nabla_{n-2}\left(x^{n-2}\right)\right) .
$$

3. If the maximum step-size is $n-1$, we have

$$
J_{n, \leq n-1}(x)=J_{n-1, \leq n-2}(x) \cdot \sum_{j=0}^{n-1} x^{j}=[n]_{x} ! .
$$

Proof. (1) This follows from the fact that a 1-step inversion is a descent. 
(3) As noted above $\operatorname{inv}_{\leq n-1}(\pi)=\operatorname{inv}(\pi)$ and therefore

$$
\begin{aligned}
J_{n, \leq n-1}(x) & =\sum_{\pi \in \mathfrak{S}_{n}} x^{\operatorname{inv}(\pi)} \\
& =(1+x)\left(1+x+x^{2}\right) \cdots\left(1+x+x^{2}+\cdots+x^{n-1}\right) \\
& =[n]_{x} ! .
\end{aligned}
$$

We also give an alternative proof: Let $k=n-1$. For each value $m$ for the last position of a permutation $\sigma$, we have that $\operatorname{inv}_{\leq k}(\sigma)=n-m+\operatorname{inv}_{\leq k}(\tau)$, where $\tau$ is the permutation obtained by flattening the restriction of $\sigma$ the domain to $\{1, \ldots, n-1\}$. Thus for each value $m$, we multiply $J_{n-1, \leq k-1}(x)$ by $x^{n-m}$ to account for all permutations that end in $m$. We then add these products together for all values of $m$ so as to account for all permutations.

(2) Let $k=n-2$. Here we imagine the effect of the first and the last positions on the inversion count of the middle positions. For each pair $\left(m_{f}, m_{\ell}\right)$ of values that the first and last positions can assume, the contribution to the counts in $J_{n, \leq k}(x)$ will be the same as their contribution of the counts in $J_{n, \leq k+1}(x)$ as long as $m_{f}<m_{\ell}$. Otherwise (if $m_{f}>m_{\ell}$ ), the contribution to $J_{n, \leq k}(x)$ is one less than it would be for $J_{n, \leq k+1}(x)$, since the first and last positions form an inversion not counted in the former, but counted in the latter.

By part (3) of this proposition, the last two factors of $J_{n, \leq k+1}(x)$ are $\left(1+x^{2}+\cdots+x^{k}\right)$ and $\left(1+x^{2}+\cdots+x^{k}+k^{k+1}\right)$, which when multiplied together give us

$$
\begin{gathered}
1+2 x+\cdots+(k-1) x^{k-2}+k x^{k-1}+(k+1) x^{k} \\
+(k+1) x^{k+1}+\cdots+3 x^{2 k-1}+2 x^{2 k}+x^{2 k+1}
\end{gathered}
$$

The coefficient of each $x^{j}$ in $(3)$ corresponds to the number of pairs $\left(m_{f}, m_{\ell}\right)$ that contribute $j$ to the inversion count of the middle positions. The number of inversions a pair $\left(m_{f}, m_{\ell}\right)$ contributes is $\left(m_{f}-1\right)$ from the first position plus $\left(n-m_{\ell}\right)$ from the last position minus possibly one for over counting in the case that $m_{f}>m_{\ell}$. So the contribution of the pair to $J_{n, \leq k+1}(x)$ would be $n+m_{f}-m_{\ell}-1$ if $m_{f}<m_{\ell}$ or $n+m_{f}-m_{\ell}-2$ if $m_{f}>m_{\ell}$. Note that if $m_{f}<m_{\ell}$, then this number is at most $n-2=k$. Otherwise (if $m_{f}>m_{\ell}$ ) this number is at least $n-1=k+1$. Thus, up to $j=k$, all pairs $\left(m_{f}, m_{\ell}\right)$ are such that $m_{f}<m_{\ell}$, and hence the exact same pairs can be used in the count for determining $J_{n, \leq k}$. Starting with $j=k+1$, all pairs $\left(m_{f}, m_{\ell}\right)$, are such that $m_{f}>m_{\ell}$, and there is an inversion counted toward $J_{n, \leq k+1}(x)$ that is not counted toward $J_{n, k}(x)$, and hence these pairs will contribute to the case where $j=k$ when constructing the formula for $J_{n, \leq k}(x)$. A similar argument shows that this generalizes, so that when $t \geq 1$, we have $x^{k+t}$ in (3) 
replaced by $x^{k+t-1}$. Thus we obtain,

$$
\begin{aligned}
J_{n, \leq k}(x)= & J_{n-1, \leq k-1}(x) \cdot\left(1+2 x+\cdots+(k-1) x^{k-2}+k x^{k-1}\right. \\
& \left.+2(k+1) x^{k}+k x^{k+1}+\cdots+2 x^{2 k-1}+x^{2 k}\right) . \\
= & J_{n-1, \leq k-1}(x) \cdot\left(\sum_{j=0}^{k}(j+1)\left(x^{j}+x^{2 k-j}\right)\right) \\
= & J_{n-1, \leq k-1}(x) \cdot\left(\sum_{j=0}^{k}(j+1) x^{j}+\sum_{j=0}^{k}(j+1) x^{2 k-j}\right) \\
= & J_{n-1, \leq k-1}(x) \cdot\left(\sum_{j=0}^{k}(j+1) x^{j}+x^{k} \sum_{j=0}^{k}(j+1) x^{k-j}\right) \\
= & J_{n-1, \leq k-1}(x) \cdot\left(\frac{\mathrm{d}}{\mathrm{d} x}\left(x \sum_{j=0}^{k} x^{j}\right)+x^{k} \nabla_{k}\left(x^{k}\right)\right) .
\end{aligned}
$$

\begin{tabular}{|c|c|c|}
\hline$n$ & $k$ & $J_{n, \leq k}(x)$ \\
\hline 1 & 1 & 1 \\
\hline 2 & 1 & $x+1$ \\
\hline \multirow{2}{*}{3} & 1 & $x^{2}+4 x+1$ \\
\hline & 2 & $(x+1)\left(x^{2}+x+1\right)$ \\
\hline \multirow{3}{*}{4} & 1 & $(x+1)\left(x^{2}+10 x+1\right)$ \\
\hline & 2 & $(x+1)\left(x^{4}+2 x^{3}+6 x^{2}+2 x+1\right)$ \\
\hline & 3 & $(x+1)\left(x^{2}+x+1\right)\left(x^{3}+x^{2}+x+1\right)$ \\
\hline \multirow{4}{*}{5} & 1 & $x^{4}+26 x^{3}+66 x^{2}+26 x+1$ \\
\hline & 2 & $(x+1)^{3}\left(x^{4}+x^{3}+11 x^{2}+x+1\right)$ \\
\hline & 3 & $(x+1)\left(x^{2}+x+1\right)\left(x^{6}+2 x^{5}+3 x^{4}+8 x^{3}+3 x^{2}+2 x+1\right)$ \\
\hline & 4 & $(x+1)\left(x^{2}+x+1\right)\left(x^{3}+x^{2}+x+1\right)\left(x^{4}+x^{3}+x^{2}+x+1\right)$ \\
\hline \multirow{5}{*}{6} & 1 & $(x+1)\left(x^{4}+56 x^{3}+246 x^{2}+56 x+1\right)$ \\
\hline & 2 & $(x+1)\left(x^{8}+4 x^{7}+25 x^{6}+88 x^{5}+124 x^{4}+88 x^{3}+25 x^{2}+4 x+1\right)$ \\
\hline & 3 & $(x+1)^{2}\left(x^{10}+3 x^{9}+7 x^{8}+22 x^{7}+31 x^{6}+52 x^{5}+31 x^{4}+22 x^{3}+7 x^{2}+3 x+1\right)$ \\
\hline & 4 & $(x+1)\left(x^{2}+x+1\right)\left(x^{3}+x^{2}+x+1\right)\left(x^{8}+2 x^{7}+3 x^{6}+4 x^{5}+10 x^{4}+4 x^{3}+3 x^{2}+2 x+1\right)$ \\
\hline & 5 & $(x+1)\left(x^{2}+x+1\right)\left(x^{3}+x^{2}+x+1\right)\left(x^{4}+x^{3}+x^{2}+x+1\right)\left(x^{5}+x^{4}+x^{3}+x^{2}+x+1\right)$ \\
\hline \multirow{6}{*}{7} & 1 & $x^{6}+120 x^{5}+1191 x^{4}+2416 x^{3}+1191 x^{2}+120 x+1$ \\
\hline & 2 & $(x+1)\left(x^{10}+5 x^{9}+39 x^{8}+218 x^{7}+562 x^{6}+870 x^{5}+562 x^{4}+218 x^{3}+39 x^{2}+5 x+1\right)$ \\
\hline & 3 & $\begin{array}{l}(x+1)\left(x^{2}+x+1\right)\left(x^{12}+4 x^{11}+10 x^{10}+38 x^{9}+79 x^{8}\right. \\
\left.+166 x^{7}+244 x^{6}+166 x^{5}+79 x^{4}+38 x^{3}+10 x^{2}+4 x+1\right)\end{array}$ \\
\hline & 4 & $\begin{array}{l}(x+1)^{4}\left(x^{2}+x+1\right) \\
\left(x^{12}+x^{11}+4 x^{10}+4 x^{9}+21 x^{8}+43 x^{6}+21 x^{4}+4 x^{3}+4 x^{2}+x+1\right)\end{array}$ \\
\hline & 5 & $\begin{array}{l}(x+1)\left(x^{2}+x+1\right)\left(x^{3}+x^{2}+x+1\right)\left(x^{4}+x^{3}+x^{2}+x+1\right) \\
\left(x^{10}+2 x^{9}+3 x^{8}+4 x^{7}+5 x^{6}+12 x^{5}+5 x^{4}+4 x^{3}+3 x^{2}+2 x+1\right)\end{array}$ \\
\hline & 6 & $\begin{array}{l}(x+1)\left(x^{2}+x+1\right)\left(x^{3}+x^{2}+x+1\right)\left(x^{4}+x^{3}+x^{2}+x+1\right) \\
\left(x^{5}+x^{4}+x^{3}+x^{2}+x+1\right)\left(x^{6}+x^{5}+x^{4}+x^{3}+x^{2}+x+1\right)\end{array}$ \\
\hline
\end{tabular}

Table 4 includes experimental runs for the distribution function $J_{n, \leq k}(x)$ for $n=$ $1, \ldots, 7$. The degree of $J_{n+1, \leq k}(x)$ is given by $\frac{k(2 n-k+1)}{2}$ since the maximum of inv $_{\leq k}$

Table 4: The distribution function of $\operatorname{inv}_{\leq k}, J_{n, \leq k}(x)$. 
is achieved by the reverse of the identity, and the number of $j$-step inversions in this permutation is $n+1-j$. Summing this number from 1 to $k$ gives the claimed degree. We would like a more complete description of the distribution function of $J_{n, \leq k}(x)$, but we leave it for future work.

\section{Future work and connections with other work}

\section{$5.1 \quad k$-step inversion tops that are zero modulo $d$}

Recall that given an inversion $(a, b)$ in a permutation, the letter $a$ is called an inversion top. Kitaev and Remmel $[4,5]$ considered inversions where the inversion top is zero modulo $d$ for a particular integer $d$. We adapt this definition to our setting by defining $\operatorname{modinv}_{d, k}(\pi)$ to be the number of $k$-step inversions with an inversion top that is zero modulo $d$. Let

$$
L_{n, d, k}(x)=\sum_{\pi \in \mathfrak{S}_{n}} x^{\operatorname{modinv}_{d, k}(\pi)}
$$

be the corresponding distribution function.

Proposition 5.1. The leading coefficient of $L_{n, 2, n-1}(x)$ is

$$
\left\lfloor\frac{n}{2}\right\rfloor^{2}(n-2) !
$$

Thus

$$
\frac{L_{n, 2, n-1}(x)}{(n-2) !}=\left\lfloor\frac{n}{2}\right\rfloor^{2} x+n(n-1)-\left\lfloor\frac{n}{2}\right\rfloor^{2} .
$$

Proof. The formula for the leading coefficient is proved as follows: In order to have one $(n-1)$-step inversion with an even inversion top, a permutation must start with an even number and end in some smaller number. Thus we get the formula

$$
(n-2) ! \sum_{j=1}^{\left\lfloor\frac{n}{2}\right\rfloor}(2 j-1) .
$$

Simplification yields the claimed formula.

We now generalize this proposition.

Proposition 5.2. Let $n / 2<k<n$ and $1<d \leq n$. The degree of $L_{n, d, k}(x)$ is $\ell=$ $\min \left(n-k,\left\lfloor\frac{n}{d}\right\rfloor\right)$ and its leading coefficient equals

$$
(n-2 \ell) ! \ell !\left(\begin{array}{c}
n-k \\
\ell
\end{array}\right) \sum_{1 \leq i_{1}<i_{2}<\cdots<i_{\ell} \leq\left\lfloor\frac{n}{d}\right\rfloor} \prod_{j=1}^{\ell}\left(d i_{j}-2 j+1\right) .
$$


Proof. Since $k>n / 2$, there are $n-k$ locations that an inversion top could be, since there are $n-k$ many $k$-step runs of length two, with the remaining $k$ many $k$-step runs being of length 1. There are $\left\lfloor\frac{n}{d}\right\rfloor$ possible values for inversion tops. Thus $\ell=\min \left(n-k,\left\lfloor\frac{n}{d}\right\rfloor\right)$ is the maximum number of inversions possible with inversion tops modulo $d$. Hence $\ell$ is the degree of $L_{n, d, k}(x)$.

The sum selects the values of the inversion tops, the $j^{\text {th }}$ smallest inversion top being $d i_{j}$. The $j^{\text {th }}$ factor of the product represents the remaining possible values that could be the bottom of the inversion with top $d i_{j}$. The positions of these inversions are chosen among the $n-k$ possible locations pairs, which is why we multiply by the binomial coefficient. The sum had arranged the tops in increasing order, and hence the coefficient of $\ell$ ! counts the ways of rearranging the tops among their $\ell$ positions. The coefficient of $(n-2 \ell)$ ! counts the ways of assigning the remaining values to the remaining positions.

Table 5 contains some empirical data for the case $d=2$.

Table 5: The distribution function of $\operatorname{modinv}_{2, k}, L_{n, 2, k}(x)$.

\begin{tabular}{|l|l|l|}
\hline$n$ & $k$ & $L_{n, 2, k}(x)$ \\
\hline 1 & 1 & 1 \\
\hline 2 & 1 & $y+1$ \\
& 2 & 2 \\
\hline & 1 & $2(y+2)$ \\
3 & 2 & $y+5$ \\
& 3 & 6 \\
\hline & 1 & $4\left(y^{2}+4 y+1\right)$ \\
4 & 2 & $2(y+1)(y+5)$ \\
& 3 & $8(y+2)$ \\
& 4 & 24 \\
\hline & 1 & $12\left(y^{2}+6 y+3\right)$ \\
& 2 & $6(y+1)(y+9)$ \\
5 & 3 & $2\left(y^{2}+22 y+37\right)$ \\
& 4 & $24(y+4)$ \\
& 5 & 120 \\
\hline & 1 & $36(y+1)\left(y^{2}+8 y+1\right)$ \\
& 2 & $4\left(4 y^{3}+55 y^{2}+94 y+27\right)$ \\
6 & 3 & $6(y+1)\left(y^{2}+22 y+37\right)$ \\
& 4 & $4(y+5)(13 y+17)$ \\
& 5 & $72(3 y+7)$ \\
& 6 & 720 \\
\hline & 1 & $144\left(y^{3}+12 y^{2}+18 y+4\right)$ \\
& 2 & $2\left(37 y^{3}+615 y^{2}+1359 y+509\right)$ \\
7 & 3 & $4\left(7 y^{3}+204 y^{2}+651 y+398\right)$ \\
& 4 & $6\left(y^{3}+75 y^{2}+387 y+377\right)$ \\
& 5 & $12\left(13 y^{2}+154 y+253\right)$ \\
& 6 & $360(3 y+11)$ \\
& 7 & 5040 \\
\hline & & \\
\hline
\end{tabular}

We would like a more complete description of the polynomial $L_{n, 2, k}(x)$, but we leave it for future work.

\subsection{Paths}

Dukes and Reifergerste [2] showed that the left boundary sum of $\pi$, written $\operatorname{lbsum}(\pi)$, is the number of inversions in $\pi$ added to the number certified non-inversions. A certified non-inversion is an occurrence of the pattern 132 which is neither part of a 1432 nor a 
1342 pattern. The mesh patterns defined by Brändén and Claesson [1] can be used to give an alternative definition: A certified non-inversions is an occurrence of the mesh pattern

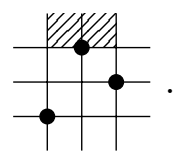

In Dukes and Reifergerste [2], the left boundary vector of a permutation $\pi$ of rank $n$ has as its $j^{\text {th }}$ coordinate the largest $i<j$, such that $\pi_{i}>\pi_{j}$. The left boundary sum of a permutation $\pi$, denoted Ibsum $(\pi)$, is defined as the sum of the left boundary coordinates. Variations of this may be as follows.

1. Define the $(\geq k)$-left boundary vector of a permutation $\pi$ to be such that its $j^{\text {th }}$ coordinate is the largest $i \leq j-k$, such that $\pi_{i}>\pi_{j}$. Define $\operatorname{Ibsum}_{\geq k}(\pi)$ to be the the sum of this vector.

2. Define the $(\leq k)$-left boundary vector of a permutation $\pi$ to be such that its $j^{\text {th }}$ coordinate is $i-\max (0, j-k)+1$, where $i$ is the largest, such that $\max (0, j-k) \leq$ $i<j$ and either $\pi_{i}>\pi_{j}$ or $i=\max (0, j-k)$. Define $\operatorname{lbsum}_{\leq k}(\pi)$ to be the sum of this vector.

3. Define the $(=k)$-left boundary vector to be such that coordinate $j$ is 1 if $a_{j-k}>a_{j}$, and 0 otherwise. Define $\operatorname{lbsum}_{=k}(\pi)$ to be the sum of this vector.

Proposition 5.3. 1. Given a permutation $\pi, \operatorname{lbsum}_{\geq k}(\pi)$ is the number of $(\geq k)$-step inversions, plus the number of non-inversions forming the end-points of an occurrence of the pattern 132, but with at least $k$ steps from the 3 to the 2 . (Such a noninversion consists of the endpoints of certified non-inversion within a permutation obtained by removing the $k-1$ positions to the left of the top of the non-inversion, and isomorphically adjusting the values to fit in the new range.)

2. Given a permutation $\pi, \operatorname{lbsum}_{\leq k}(\pi)$ is the number of $(\leq k)$-step inversions plus the number of certified $(\leq k)$-step non-inversions (a certified non-inversions whose endpoints are at most $k$ apart).

3. Given a permutation $\pi, \operatorname{Ibsum}_{=k}(\pi)$ is the number of $k$-step inversions of $\pi$.

Proof. The proof of these are adapted from [2].

1. Let $\left(a_{1}, \ldots, a_{n}\right)$ be the $(\geq k)$-left boundary vector. Then $a_{j}=d$ is the maximum value no less than $k$ away from $j$, such that $\pi_{d}>\pi_{j}$. Then every $i \leq d$ is such that either $\pi_{i}>\pi_{j}$ or there is a position $c$ (we can always choose $d$ ), such that $i<c \leq j-k$ and $\pi_{c}>\pi_{j}$. Since $d$ is maximal, every $(\geq k)$-step inversion with bottom $j$ will be counted among such $i$, and no $(<k)$-step inversion will qualify, since $d$ is already $k$-steps away. Furthermore, any $(\geq k)$-step non-inversion $(i, j)$ with $i<d$, such that there is a $c$ (we can always pick $d$ ), such that $i<c \leq j-k$ and $\pi_{c}>\pi_{j}$. Because $d$ is maximal, any non-inversion $(i, j)$ that has a $c$, such that $i<c \leq j-k$ and $\pi_{c}>\pi_{j}$, is a non-inversion, where $i<d$. 
2. The proof here is almost identical to the previous case, except we restrict $d$ to ranging from $\max (0, j-k)$ to $j-1$, and we only consider $i \leq d$, such that $i \geq j-k$. This allows us to focus on $(\leq k)$-step inversions and certified $(\leq k)$-step non-inversions, and is accounted for by the fact that the $a_{j}$ is really $d-\max (0, j-k)+1$. Furthermore, since we are technically counting certified non-inversions, which are triples rather than pairs, we select for the middle point the position of maximum value. If we did not place this restriction, we could over-count, with many possibilities for a middle, given one pair of endpoints.

3. The coordinates of the $(=k)$-left boundary vector with the value 1 are precisely the positions of the permutation that form the top of a $k$-step non-inversion. Since a position can be the top of at most one $k$-step non-inversion, the sum of the coordinates of the vector is equal to the number of $k$-step non-inversions.

We consider yet a forth way to generalize $\operatorname{Ibsum}(\pi)$. For our purposes we define a certified $k$-step non-inversion to be a certified non-inversion with endpoints forming a $k$-step non-inversion. The generalization we focus on from here is as follows. For a permutation $\pi$, let ipcni ${ }_{k}(\pi)$ be the number of $k$-step inversions in $\pi$ added to the number of certified $k$-step non-inversions. Let

$$
K_{n, k}(x)=\sum_{\pi \in \mathfrak{S}_{n}} x^{\mathrm{ipcni}_{k}(\pi)}
$$

be the corresponding distribution function.

From the empirical data in Table 6 it seems that the constant term in $K_{n, k}(x)$ is always equal to $k$ !. This is proven below.

Proposition 5.4. The constant term in $K_{n, k}$ is $k$ !. Furthermore, the permutations $\pi \in$ $\mathfrak{S}_{n}$, such that $\operatorname{ipcni}_{k}(\pi)=0$, are precisely the permutations that have the form $\sigma(k+1)(k+$ 2) $\cdots n$, where $\sigma \in \mathfrak{S}_{k}$.

Proof. It is clear that any permutation of the form $\sigma(k+1)(k+2) \cdots n$, where $\sigma$ is a permutation from $S_{k}$ has ipcni ${ }_{k}$ zero. Conversely, suppose that ipcni ${ }_{k}(\pi)=0$ and that $\pi$ is of the form $\sigma \lambda$ where $\sigma$ consists of the first $k$ letters of $\pi$ and $\lambda$ consists of the remaining letters. Then the letters of $\lambda$ must be in increasing order; otherwise we let $\ell_{1}>\ell_{2}$ be the first two adjacent letters in $\lambda$ that are not in increasing order. Then if $\pi_{\ell_{2}-k}<\pi_{\ell_{2}}$, there is an $\ell$, such that the triple $\left(\ell_{2}-k, \ell, \ell_{2}\right)$ is a certified $k$-step non-inversion, and if $\pi_{\ell_{2}-k}>\pi_{\ell_{2}}$, the pair $\left(\ell_{2}-k, \ell_{2}\right)$ is an inversion. Now to finish the proof we need to show that $\sigma$ consists of the letters $1, \ldots, k$. First observe that the first letter of $\lambda$ is larger than any letter in $\sigma$. Since $\lambda$ is increasing, the remaining letters in $\lambda$ are also larger than $1, \ldots, k$. This finishes the proof.

Corollary 5.5. The number of permutations $\pi \in \mathfrak{S}_{n}$ with ipcni $_{n-1}(\pi)=1$ is $(n-1)(n-$ 1)!. Thus

$$
\frac{K_{n, n-1}(x)}{(n-1) !}=(n-1) x+1
$$


Table 6: The distribution function of $\mathrm{ipcni}_{k}, K_{n, k}(x)$.

\begin{tabular}{|l|l|l|}
\hline$n$ & $k$ & $K_{n, k}(x)$ \\
\hline 1 & 1 & 1 \\
\hline 2 & 1 & $x+1$ \\
& 2 & 2 \\
\hline 3 & 1 & $x^{2}+4 x+1$ \\
& 2 & $2(2 x+1)$ \\
& 3 & 6 \\
\hline 4 & 1 & $(x+1)\left(x^{2}+10 x+1\right)$ \\
& 2 & $2(x+1)(5 x+1)$ \\
& 3 & $6(3 x+1)$ \\
\hline 5 & 4 & 24 \\
& 1 & $x^{4}+26 x^{3}+66 x^{2}+26 x+1$ \\
& 2 & $2\left(13 x^{3}+35 x^{2}+11 x+1\right)$ \\
& 3 & $6\left(11 x^{2}+8 x+1\right)$ \\
& 4 & $24(4 x+1)$ \\
\hline 6 & 5 & 120 \\
& 1 & $(x+1)\left(x^{4}+56 x^{3}+246 x^{2}+56 x+1\right)$ \\
& 2 & $2\left(38 x^{4}+183 x^{3}+121 x^{2}+17 x+1\right)$ \\
& 3 & $6(x+1)\left(46 x^{2}+13 x+1\right)$ \\
& 4 & $24\left(19 x^{2}+10 x+1\right)$ \\
& 5 & $120(5 x+1)$ \\
& 6 & 720 \\
\hline 7 & 1 & $x^{6}+120 x^{5}+1191 x^{4}+2416 x^{3} 119 x^{2}+120 x+1$ \\
& 2 & $2\left(116 x^{5}+969 x^{4}+1100 x^{3}+310 x^{2}+24 x+1\right)$ \\
& 3 & $6\left(202 x^{4}+459 x^{3}+157 x^{2}+21 x+1\right)$ \\
& 4 & $24\left(103 x^{3}+89 x^{2}+17 x+1\right)$ \\
& 5 & $120\left(29 x^{2}+12 x+1\right)$ \\
& 6 & $720(6 x+1)$ \\
\hline 8 & 7 & 5040 \\
& 1 & $(x+1)\left(x^{6}+246 x^{5}+4047 x^{4}+11572 x^{3}+4047 x^{2}+246 x+1\right)$ \\
& 2 & $2\left(382 x^{6}+5124 x^{5}+9517 x^{4}+4420 x^{3}+684 x^{2}+32 x+1\right)$ \\
& 3 & $6\left(986 x^{5}+3454 x^{4}+1925 x^{3}+325 x^{2}+29 x+1\right)$ \\
& 4 & $24(x+1)\left(614 x^{3}+201 x^{2}+24 x+1\right)$ \\
& 5 & $120\left(190 x^{3}+125 x^{2}+20 x+1\right)$ \\
& 6 & $720\left(41 x^{2}+14 x+1\right)$ \\
& 7 & $5040(7 x+1)$ \\
& 8 & 40320 \\
& & \\
\hline &
\end{tabular}

Proof. As a consequence of Proposition 5.4, given $\pi \in \mathfrak{S}_{n}$, ipcni ${ }_{n-1}(\pi)=0$ if and only if $\pi_{n}=n$. Note that $\operatorname{ipcni}_{k}(\pi)=1$ otherwise. There are $(n-1)(n-1)$ ! permutation $\pi$, such that $\pi_{n} \neq n$.

Proposition 5.6. The number of permutations $\pi \in \mathfrak{S}_{n}$, with ipcni $_{n-2}(\pi)=2$ is

$$
(n-2) !\left(n^{2}-3 n+1\right) \text {. }
$$

Thus

$$
\frac{K_{n, n-2}(x)}{(n-2) !}=\left(n^{2}-3 n+1\right) x^{2}+2(n-1) x+1 .
$$

Proof. In order to construct a permutation with ipcni ${ }_{n-2}$ equal to 2 we need to choose four numbers to occupy the first two positions and the last two positions. The permutation constructed in this way will always have ipcni ${ }_{n-2}$ equal to 2 unless any of the following hold:

- $n$ is in position 1 and $n-1$ is in position $n$,

- $n$ is in position $n-1$, or 
- $n$ is in position $n$.

This shows that the number we are looking for is

$$
(n-4) !(n(n-1)(n-2)(n-3)(n-4)-(n-2)(n-3)-2(n-1)(n-2)(n-3)) .
$$

When this is simplified, it gives the formula in the proposition.

\subsection{Marked mesh patterns}

Marked mesh patterns were defined by Úlfarsson in [7, Definition 24]. In this subsection we show how these patterns relate to the concepts introduced above. Figure 2 shows how $k$-step, $(\leq k)$-step and $\left(k_{1}, k_{2}\right)$-step inversions can be identified with patterns.
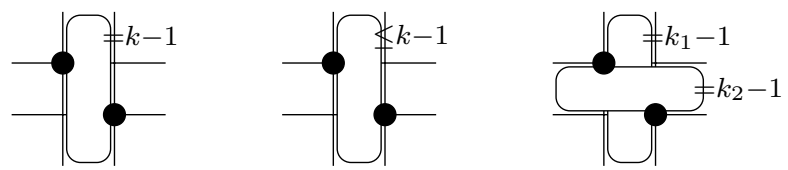

Figure 2: $k$-step, $(\leq k)$-step and $\left(k_{1}, k_{2}\right)$-step inversions in terms of patterns.

Using the representation of $k$-step inversions allows us to write the number of inversions and the inversion sum of a permutation as a linear combination of patterns; see Figure 3.

$$
\operatorname{inv}=\sum_{k \geq 1}(\overbrace{k \geq 1}^{\# k-1}), \quad \text { invsum }=\sum_{k} k \cdot(
$$

Figure 3: Writing the inv and invsum as a linear combination of patterns.

It is only slightly harder to realize that the coordinates of the zone-crossing vectors are given by patterns, for example, the $k^{\text {th }}$ coordinate of the inversion zone-crossing vector of a permutation $\pi$ is the number of occurrences of the pattern

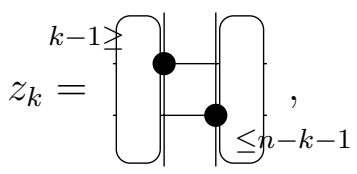

in $\pi$. A $k$-step inversion with an inversion top that is zero modulo $d$ is an occurrence of

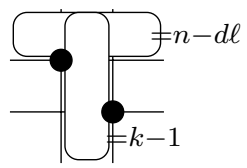


for some $\ell \geq 1$.

Finally, a certified $k$-step non-inversion is an occurrence of the pattern

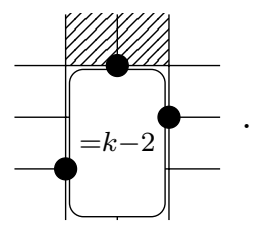

\section{Acknowledgements}

We would like to thank Anders Claesson and Einar Steingrímsson for their helpful comments. We would also like to thank an anonymous referee for detailed and helpful comments.

\section{References}

[1] Petter Brändén and Anders Claesson, Mesh patterns and the expansion of permutation statistics as sums of permutation patterns, in preparation.

[2] Mark Dukes and Astrid Reifegerste, The area above the Dyck path of a permutation, Adv. in Appl. Math. 45 (2010), no. 1, 15-23. MR 2628782

[3] Fredrik Jansson, Variations on the excedance statistic in permutations, Master's thesis, Chalmers Tekniska Högskola, 2006.

[4] Sergey Kitaev and Jeffrey Remmel, Classifying descents according to equivalence mod $k$, Electron. J. Combin. 13 (2006), no. 1, Research Paper 64, 39 pp. (electronic). MR 2240770 (2007b:05007)

[5] _ Classifying descents according to parity, Ann. Comb. 11 (2007), no. 2, 173193. MR 2336014 (2008f:05008)

[6] Percy A. MacMahon, Combinatory analysis. Vol. I, II (bound in one volume), Dover Phoenix Editions, Dover Publications Inc., Mineola, NY, 2004, Reprint of An introduction to combinatory analysis (1920) and Combinatory analysis. Vol. I, II (1915, 1916). MR 2417935 (2009e:05002)

[7] Henning Úlfarsson, A unification of permutation patterns related to Schubert varieties, Pure Math. Appl. to appear (2011). 